\title{
Identificación de corredores prioritarios de carreteras para la aplicación de estrategias de reducción de emisiones GEI. Caso de España.
}

\author{
Natalia Sobrino Vázquez \\ Doctora Ingeniera Civil, TRANSyT-UPM \\ Andrés Monzón de Cáceres \\ Catedrático de Transporte, UPM
}

\section{RESUMEN}

Lograr un transporte bajo en carbono es crucial en la planificación y gestión del transporte por carretera. Existen diversas acciones para la reducción de emisiones del transporte por carretera: sobre la demanda, la tecnología del vehículo, la gestión del tráfico, etc. Pero, antes de realizar cualquier actuación, es necesario identificar cuáles son los puntos de la red que necesitan una acción prioritaria.

Este trabajo describe un índice para la identificación de tramos y corredores prioritarios para hacer frente a los problemas de emisiones GEI. La herramienta HERA de evaluación del consumo de energía y emisiones del tráfico, es utilizada para estimar los parámetros del Índice Prioritario de Emisiones (IPE) y para evaluar diferentes estrategias de gestión y diseño de carreteras. El IPE identifica la prioridad de acción en un tramo o corredor en base a las emisiones totales del tráfico y a la alta intensidad de emisiones unitarias del tramo, es decir, la ineficiencia.

La Red de Carreteras del Estado (2012) se toma como caso de estudio, y los resultados se muestran en mapas ayudados por un SIG. Se identifican siete corredores prioritarios, que representan el $25 \%$ de la red y contribuyen con el $52 \%$ de las emisiones GEI totales de la red en 2012. Los corredores más ineficientes corresponden a aquellos con una alta proporción de vehículos pesados, altas velocidades de vehículos ligeros y aquellos que presentan pendientes pronunciadas. Sobre los corredores prioritarios han sido aplicadas una serie de estrategias de reducción de emisiones. La estrategia más efectiva -aparte de la integración de varias estrategias- es la reducción de la velocidad de los vehículos ligeros. Una reducción de velocidad de $-10 \mathrm{~km} / \mathrm{h}$ podría producir un $3,85 \%$ de ahorro anual de emisiones sobre dichos corredores, llegando a ahorrar $566 \mathrm{KtCO}_{2} \mathrm{eq}$ anuales.

\section{INTRODUCCIÓN}

El transporte por carretera es uno de los sectores prioritarios para la aplicación de estrategias de reducción de emisiones de gases de efecto invernadero (GEI) (SIMS et al., 2014) ya que es la segunda mayor fuente de emisiones GEI en la UE, representado el 18\% de dichas emisiones totales en 2012 (AEMA, 2014). En 2011, España fue el quinto país de Europa con mayor contribución de emisiones del transporte por carretera con 80,4 millones de toneladas de $\mathrm{CO}_{2}$, después de Alemania, Francia, Reino Unido e Italia (EUROSTAT, 2014). Por ello, lograr un transporte por carretera bajo en carbono es crucial en la planificación y gestión del 
transporte por carretera. Para ello, en España se vienen aplicando medidas de eficiencia energética para el transporte por carretera en los diferentes Planes de Acción de Ahorro y Eficiencia Energética 2004-2012 y 2011-2020 (IDAE, 2003, 2011). El objetivo de este último es reducir el consumo total de energía nacional en un $20 \%$ en 2020 , de conformidad con la estrategia europea 2020 de energía (COM, 2010). Un tercio de dicho objetivo debe lograrse mediante el ahorro en el sector del transporte por carretera (IDAE, 2011). Las estrategias a aplicar se dividen en dos grandes grupos, por un lado acciones para reducir el uso del transporte por carretera en general (por ejemplo, cambio modal); y por otro lado acciones para mejorar la eficiencia energética y de carbono del tráfico rodado (por ejemplo, estrategias de gestión de tráfico y renovación de flotas). La gestión eficiente del sistema de transporte por carretera persigue estrategias que reducen el consumo energético y el ratio de $\mathrm{CO}_{2}$ eq (BANISTER et al. 2011), en definitiva, son estrategias de reducción del consumo energético del tráfico en la fase de operación (gestión de velocidad, de vehículos pesados, rutas eficientes, etc.) y estrategias sobre la infraestructura (mejora de la infraestructura existente o nueva infraestructura) para reducir el consumo energético y las emisiones en la fase de explotación de la carretera.

En la definición de estos planes de acción y descripción de estrategias, las autoridades competentes necesitan, en primer lugar, metodologías para clasificar y priorizar aquellos tramos o corredores de la red con mayores problemas de consumo energético y emisiones GEI, sobre los que se aplicaran las acciones de eficiencia energética y bajo carbono. Por lo que las cuestiones que se plantean son: ¿Cómo pueden ser diseñados dichos planes de acción para reducir las emisiones de tráfico? ¿Cuáles son los tramos o corredores prioritarios de la red en estudio? ¿Cuánto de eficientes son las estrategias de gestión del sistema de transporte?

En el presente artículo se describe un índice para la identificación de tramos y corredores prioritarios (IPE) para hacer frente a los problemas de emisiones GEI en la red de carreteras.

\section{LA METODOLOGÍA HERA: Huella Energética de opeRación de Autopistas}

\subsection{Evaluación de emisiones del tráfico de autopistas: HERA}

La herramienta HERA ${ }^{1}$ es una metodología utilizada para evaluar la huella energética y de carbono del tráfico rodado de un tramo, vía de alta capacidad o red (SOBRINO et al., 2014). HERA permite estimar el consumo de combustible, consumo energético y emisiones GEI del tráfico de una red, vía o tramo en la escala temporal de un año, bajo unas condiciones de tráfico e infraestructura determinadas. HERA se basa en una metodología bottom-up que combina para cada tipología de vehículo un modelo de consumo energético basado en la velocidad media y ajustado con la pendiente del tramo en estudio, COPERT IV (GKATZOFLIAS et al. 2007). Las principales características de HERA se muestran en la Figura 1. En primer lugar el modelo es calibrado para cada caso de estudio según la

1 http://www.hera.transyt.upm.es/ 
composición de la flota de vehículos, seguidamente la red o carretera de estudio es divida en tramos homogéneos de velocidad, intensidad de tráfico y pendiente que son los datos de entrada en el modelo. Para cada tramo, utilizando el modelo HERA se obtienen los resultados de consumo de combustible (toneladas de fuel/año), consumo energético (MJ/año) y emisiones GEI (toneladas de $\mathrm{CO}_{2}$ eq/año); y también la intensidad energética y de emisiones por cada tramo (MJ/veh-km; $\mathrm{CO}_{2} \mathrm{eq} / \mathrm{veh}-\mathrm{km}$, respectivamente). La intensidad energética y de emisiones está fuertemente relacionada con las características del tráfico (por ejemplo la presencia de pesados) y el comportamiento de conducción (por ejemplo altas velocidades). Con la evaluación de los resultados, se pueden proponer políticas de eficiencia energética que actúen sobre los parámetros de entrada (velocidad, tráfico, alineación horizontal, etc.) y comprobar su eficiencia en comparación con los resultados de partida.

Las principales contribuciones de HERA con respecto a otras herramientas ya existentes (COPERT, ARTEMIS, etc.) para el cálculo de emisiones de tráfico son: que tiene un enfoque tramo a tramo; que el modelo se calibra para cada área o red de estudio según la composición de la flota de vehículos, Y que tiene en cuenta el efecto de la alineación horizontal en el consumo energético y emisiones (rampas/pendientes) como se ha probado en diversos estudios su influencia (ejemplo: HERNANDEZ et al. 2013),

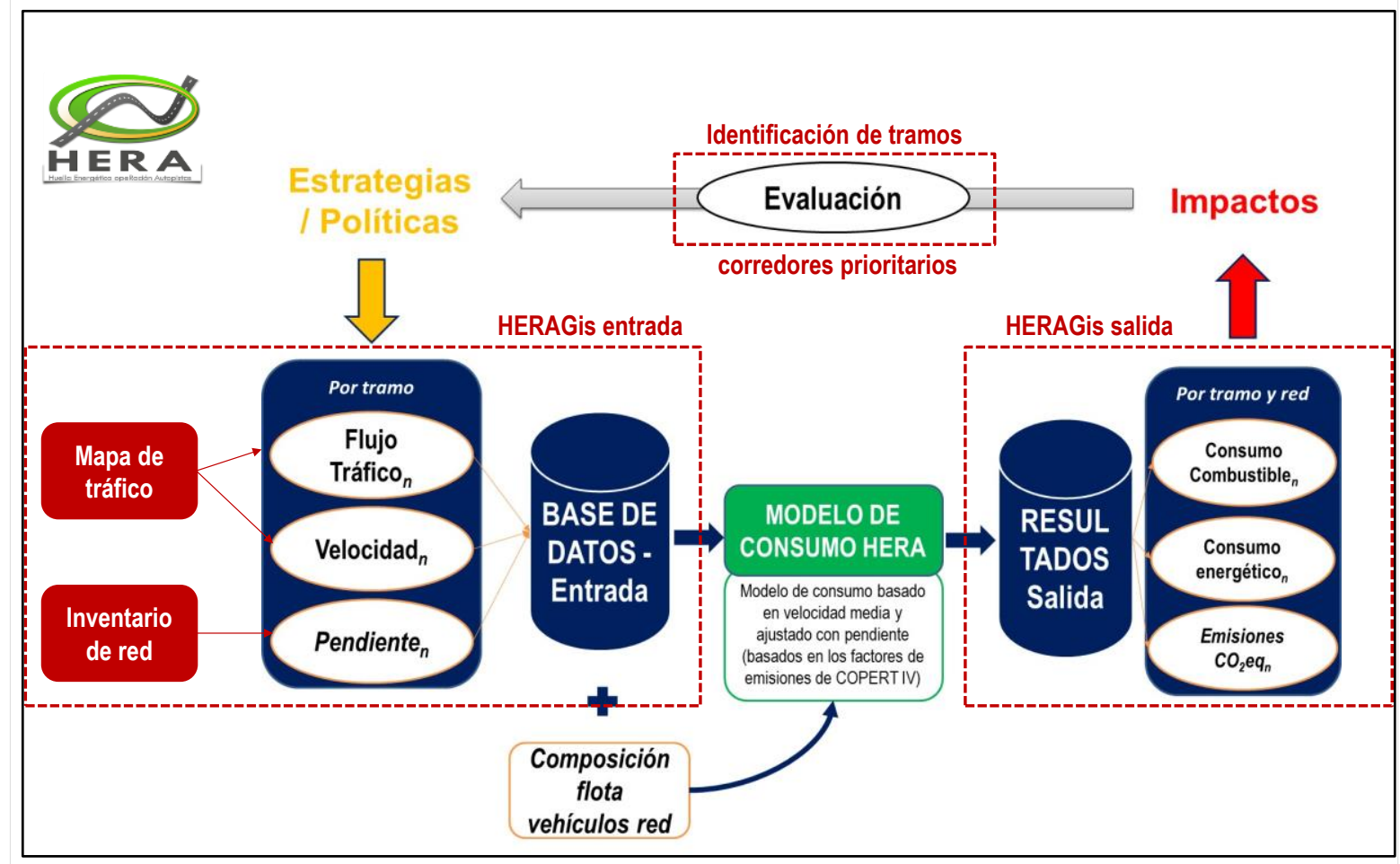

Fig. 1 - Marco metodológico de HERA y su interfaz con datos georreferenciados

\subsection{Interfaz HERA con datos georreferenciados: HERAGis}

Otra de las contribuciones de HERA es que permite conectar la entrada y salida de datos con un Sistema de Información Geográfica (SIG) proporcionando una representación gráfica del consumo de energía y las emisiones GEI de la red. De esta forma, tal y como se muestra en granate en la Figura 1, se pueden utilizar Mapas de Tráfico de la red para obtener tramo a 
tramo los datos de velocidad media por tipo de vehículo y la intensidad media de tráfico diario (IMD), con cada tramo georreferenciado; y unirlo con los datos geométricos de los tramos que se obtienen de Mapas de Inventarios de la red de carreteras, formando así HERAGis entrada. Utilizando HERA se obtiene HERAGis resultados por tramo, que pueden ser representados en forma de mapa, identificando de esta forma los tramos con más emisiones y más ineficientes.

\section{IDENTIFICACIÓN DE TRAMOS PRIORITARIOS: Índice Prioritario de Emisiones}

A partir de los resultados georreferenciados, a continuación se describe un procedimiento para la identificación de tramos prioritarios de actuación basados en el cálculo de un índice denominado IPE: Índice Prioritario de Emisiones, de forma similar al procedimiento de RUIZ-PADILLO et al. (2014) para identificar impactos de ruido.

El procedimiento de cálculo del IPE consiste en 4 pasos:

Paso 1. Selección de los parámetros que representen el índice. Ellos son: las emisiones GEI totales (ET) por tramo (expresadas en $\mathrm{CO}_{2} \mathrm{eq}$ ) y la de intensidad de emisiones (IE) por tramo $\left(\mathrm{CO}_{2} \mathrm{eq} / \mathrm{veh}-\mathrm{km}\right)$. Las emisiones totales representan las emisiones producidas por el total del tráfico que transita por la carretera, mientras que la intensidad de emisión sirve para identificar los tramos más contaminantes basados en la forma de operación (velocidad, tipología de vehículos, etc.). De esta forma el índice IPE tiene en cuenta tanto el tráfico total como las condiciones de operación de la carretera.

Paso 2. Transformación de los parámetros en indicadores utilizando las funciones de transformación y que están basadas en el método SIG para la clasificación de datos basados en la desviación estándar de los resultados de todos los segmentos de la red de estudio (en intervalos de 0,$25 ; 0,5 ; 0,75$ y 1). La Figura 2 muestra un ejemplo de cómo calcular las funciones de transformación en base a los resultados de la desviación estándar en SIG. Emisiones Totales (ET) e Intensidad de Emisión (IE), cuyos rangos van de 0 a 1.

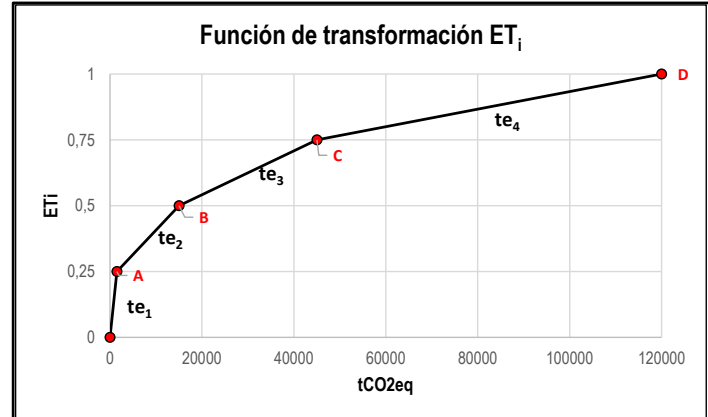

\begin{tabular}{|c|c|c|}
\hline Emisiones totales & \multicolumn{2}{|c|}{ Función Transformación ET } \\
\hline Si $x_{i} \leq \mathrm{A}$ tCO2eq & $\mathrm{te}_{1}$ & $\mathrm{ET}_{\mathrm{i}}=a \mathrm{x}_{\mathrm{i}}$ \\
\hline Si $A<x_{i} \leq B$ tCO2eq & $\mathrm{te}_{2}$ & $E T_{i}=\beta+y x_{i}$ \\
\hline Si B $<x_{i} \leq C$ tCO2eq & $\mathrm{te}_{2}$ & $E T_{i}=\delta+\varepsilon x_{i}$ \\
\hline Si $x_{i} \geq C$ tCO2eq & $\mathrm{te}_{2}$ & $E T_{i}=\eta+\theta x_{i}$ \\
\hline
\end{tabular}

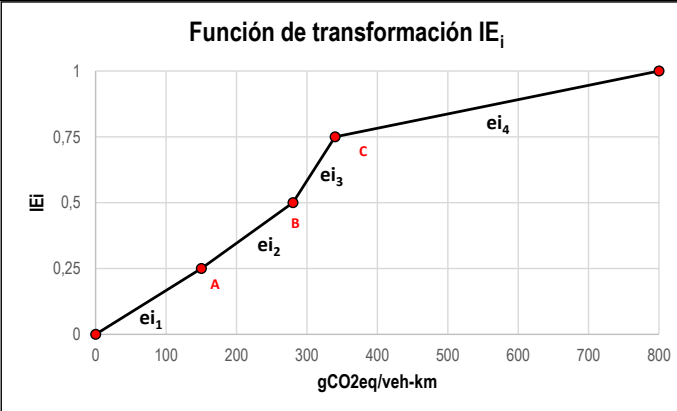

\begin{tabular}{|c|c|c|}
\hline Intensidad de Emisiones & \multicolumn{2}{|c|}{ Función Transformación $\mathrm{EI}_{\mathrm{i}}$} \\
\hline Si $x_{i} \leq \mathrm{A}$ gCO2eq/veh-km & te $_{1}$ & $E l_{i}=\alpha x_{i}$ \\
\hline Si $\mathrm{A}<\mathrm{x}_{i} \leq \mathrm{B}$ gCO2eq/veh-km & $\mathrm{te}_{2}$ & $E l_{i}=\beta+\gamma x_{i}$ \\
\hline Si B $<x_{i} \leq$ C gCO2eq/veh-km & $\mathrm{te}_{2}$ & $E \mathrm{l}_{\mathrm{i}}=\delta+\varepsilon \mathrm{x}_{\mathrm{i}}$ \\
\hline Si $x_{i} \geq$ C gCO2eq/veh-km & $\mathrm{te}_{2}$ & $E \mathrm{I}_{\mathrm{i}}=\eta+\theta \mathrm{x}_{\mathrm{i}}$ \\
\hline
\end{tabular}

Fig. 2 - Funciones de transformación a partir de: emisiones totales (TE) e intensidad de emisiones (EI). 
Paso 3. El IPE se obtiene como combinación de los dos indicadores:

- Tramo no prioritario cuando: ET y IE < 0,5;

- Tramo con prioridad alta cuando: ET o IE $\geq 0,5$

- Tramo con prioridad muy alta cuando: ET y IE > 0.5

Paso 4. En base al cálculo del IPE para cada tramo, un corredor es prioritario cuando al menos la mitad de su longitud tiene un IPE con prioridad alta o muy alta.

\section{CASO DE ESTUDIO: RED DE CARRETERAS DEL ESTADO}

\subsection{Emisiones GEI e Intensidad GEI de los tramos de la RCE en 2012}

Se toma como caso de estudio la Red de Carreteras del Estado, RCE. La RCE representa el $15.7 \%$ de la red total de carreteras de España, 165.595km (MF, 2013). La RCE contiene autopistas de peaje, autovías, carreteras nacionales y vías de alta capacidad metropolitanas. Del tráfico total en España, el 51.6\% pertenece a la RCE con el $63.1 \%$ del tráfico pesado. En consecuencia, la RCE ofrece una descripción muy aproximada del tráfico interurbano de España.

Para el análisis de la RCE se toman como datos de entrada, las características de la flota de vehículos a partir de las series estadísticas de la DGT (DGT, 2013), el Mapa de Tráfico de 2012 (MF, 2013) y el Inventario de Carreteras de España (MF, 2010). Con la unión de forma vectorial de dichos datos en SIG, se obtiene una red de $26.038 \mathrm{~km}$ de longitud dividida en 4.778 tramos homogéneos (siendo la media de longitud de los tramos de $5.44 \mathrm{~km}$ ).

\begin{tabular}{|l|r|r|r|r|r|}
\hline \multicolumn{1}{|c|}{ Tipo carretera } & \multicolumn{1}{|c|}{$\begin{array}{c}\text { Autopista de } \\
\text { Peaje }\end{array}$} & \multicolumn{1}{c|}{$\begin{array}{c}\text { Autovía } \\
\text { Libre }\end{array}$} & $\begin{array}{c}\text { Carretera } \\
\text { Nacional 1 } \\
\text { nivel }\end{array}$ & $\begin{array}{c}\text { Autovía } \\
\text { metropolitana }\end{array}$ & Total \\
\hline Longitud (km) & 2.548 & 7.858 & 14.733 & 881 & $\mathbf{2 6 . 0 2 0}$ \\
\hline IMD (veh/día) & 15.858 & 21.489 & 4.480 & 42.523 & $\mathbf{1 2 . 0 1 9}$ \\
\hline$\%$ de tráfico pesado & $12.04 \%$ & $17,01 \%$ & $13,19 \%$ & $8,69 \%$ & $\mathbf{1 4 , 0 8 \%}$ \\
\hline $\begin{array}{l}\text { Velocidad media } \\
\text { vehículos ligeros }\end{array}$ & 106 & 110 & 231,6 & 221,7 & $\mathbf{9 3}$ \\
\hline $\begin{array}{l}\text { Intensidad de emisión } \\
\text { media (gCO } 2 \text { eq/veh- } \\
\text { km) }\end{array}$ & 258,5 & 275,2 & & 247 \\
\hline $\begin{array}{l}\text { Emisiones Totales } \\
\text { (KtCO }{ }_{2} \text { eq) }\end{array}$ & $3.882,7$ & $15.804,1$ & $5.554,8$ & $2.892,7$ & $\mathbf{2 8 . 1 3 4 , 3}$ \\
\hline$\%$ emisiones totales & $13.81 \%$ & $56,17 \%$ & $19,74 \%$ & $10,28 \%$ & \\
\hline
\end{tabular}

Tabla 1 - Resumen de las especificaciones sobre fuentes

Utilizando HERA se obtiene que en 2012, la RCE emitió alrededor de 28 millones de toneladas de $\mathrm{CO}_{2}$ eq. Esta cantidad total representa el 37.65\% del total de las emisiones del transporte por carretera en España (incluyendo red autonómica, provincial y local). De esos 
28 millones de toneladas, más de la mitad se producen en las autovías, coincidiendo así con tramos con una alta proporción de vehículos pesados (17\% del volumen del tráfico) y con altas velocidades de los vehículos ligeros $(110 \mathrm{~km} / \mathrm{h}$ de media). En cuanto a la intensidad de emisión, cuanto más pequeña es la intensidad, más eficiente es el tramo. La intensidad de emisión media por tramo son 247 gramos de $\mathrm{CO}_{2}$ eq por vehículo y kilometro con un volumen de tráfico de pesados del $14 \%$ y una velocidad media de los vehículos ligeros de $93 \mathrm{~km} / \mathrm{h}$. Como en el caso anterior, las autovías son las que presentan mayor intensidad de emisiones con 275 gramos de $\mathrm{CO}_{2}$ eq por vehículo y kilómetro de media.

\subsection{Cálculo de IPE e identificación de corredores prioritarios de la RCE}

Aplicando el procedimiento de cálculo del Índice Prioritario de Emisiones, IPE, se identifican en la siguiente Figura 3 los siete corredores prioritarios de la RCE. Estos corredores conectan las ciudades principales como Barcelona, Sevilla o Bilbao con la capital, Madrid. Sin embargo, también aparece como prioritario el corredor del Mediterráneo dada la importancia de la conexión del transporte entre Francia y el Sur de España por la costa.

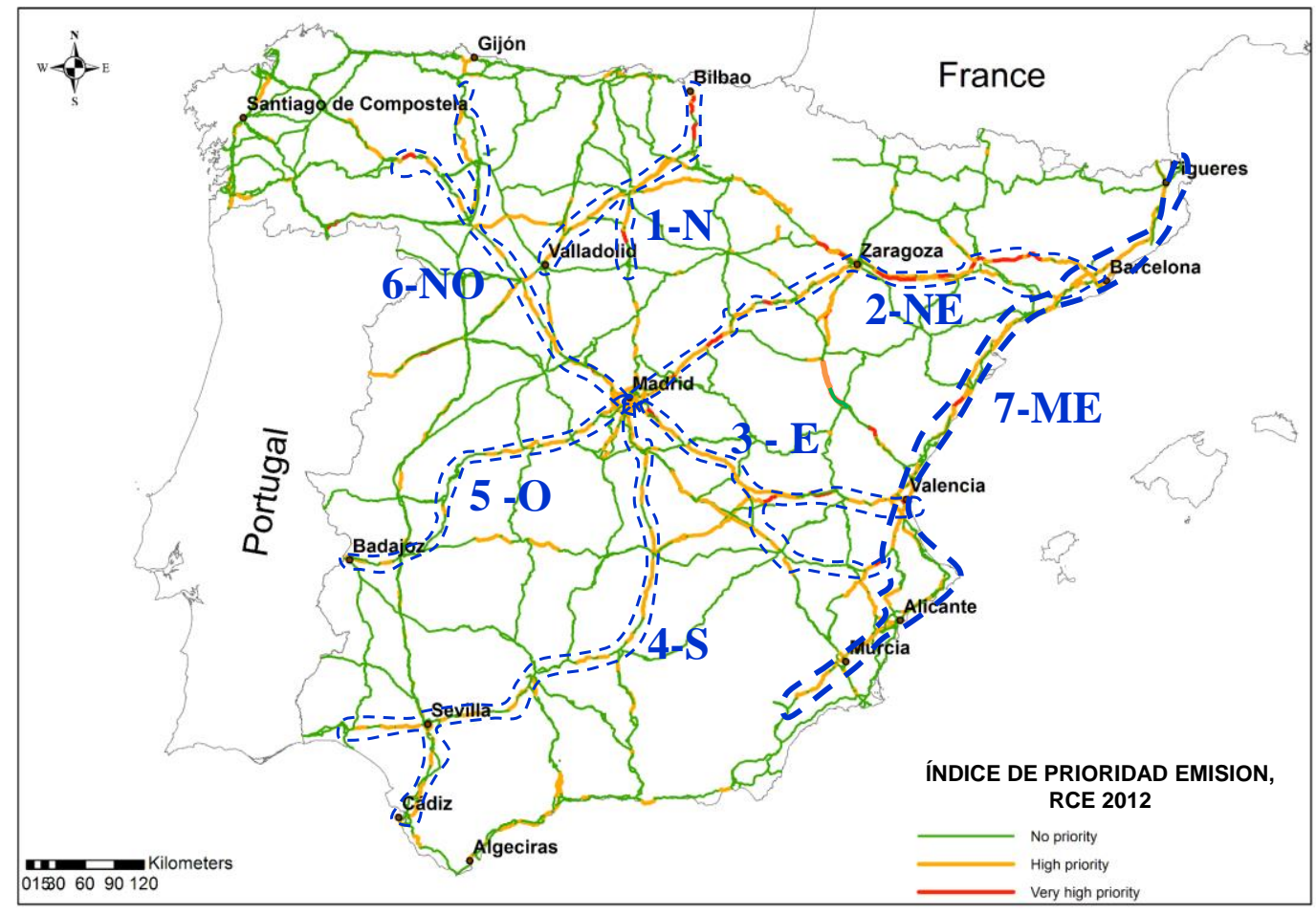

Fig. 3 - Identificación de los corredores prioritarios de la RCE en 2012 basado en IPE

La siguiente Tabla 2 contiene un resumen de los resultados principales para cada corredor. Se han identificado cuatro tipos de características de las carreteras para agrupar los corredores: cuando el corredor tiene dos alternativas (libre de pago y pago), cuando es un corredor principal (alto volumen de tráfico), cuando tiene gran volumen de pesados, y cuando tiene grandes desniveles por pasos de montaña.

Los siete corredores prioritarios son responsables del 51\% del total de emisiones de la RCE, aunque solo representan el $25 \%$ de la longitud de la red. El corredor con más emisiones es el Mediterráneo (7), que contribuye con el 16,85\% de las emisiones de la RCE. Es el corredor 
más largo y con más volumen de tráfico. El corredor más ineficiente en términos de emisiones es el Corredor Noreste (2), con una intensidad de emisiones de 315 gramos de $\mathrm{CO}_{2}$ eq por vehículo y kilómetro. Esto se debe al gran volumen de tráfico (24.394 vehículos diarios) y un alto porcentaje de vehículos pesados $(23,4 \%)$. Por lo tanto, los corredores ineficientes corresponden aquellos que tienen un alto porcentaje de vehículos pesados. Sobre las áreas montañosas, el corredor del Noroeste (6) tiene las pendientes más pronunciadas ( $11 \%$ de la longitud del corredor tiene desniveles mayores al 2\%). El corredor 6 contribuye con el 4,64\% de las emisiones GEI de la RCE.

\begin{tabular}{|c|c|c|c|c|c|c|c|c|}
\hline & $\begin{array}{c}1 . \\
\text { Corredo } \\
\text { r Norte } \\
\text { N }\end{array}$ & $\begin{array}{c}2 . \\
\text { Corredor } \\
\text { Noreste } \\
\text { NE }\end{array}$ & $\begin{array}{c}3 . \\
\text { Corredor } \\
\text { Este } \\
\text { E }\end{array}$ & $\begin{array}{c}4 . \\
\text { Corredor } \\
\text { Sur } \\
\text { S }\end{array}$ & $\begin{array}{c}5 . \\
\text { Corredor } \\
\text { Oeste } \\
\text { O }\end{array}$ & $\begin{array}{c}6 . \\
\text { Corredor } \\
\text { Noroeste } \\
\text { NO }\end{array}$ & $\begin{array}{c}7 . \\
\text { Corredor } \\
\text { Medite- } \\
\text { rraneo } \\
\text { ME }\end{array}$ & Total \\
\hline Longitud(km) & 487 & 1.054 & 736 & 999 & 535 & 938 & 1.788 & 6.537 \\
\hline $\mathrm{IMD}(\mathrm{veh} / \mathrm{km})$ & 14.931 & 24.394 & 21.431 & 25.182 & 19.847 & 15.113 & 27.865 & \\
\hline$\%$ pesados & $23,76 \%$ & $23,42 \%$ & $20,95 \%$ & $16.04 \%$ & $12,73 \%$ & $14,55 \%$ & $15.81 \%$ & \\
\hline $\begin{array}{l}\text { Velocidad } \\
\text { media ligeros } \\
(\mathrm{km} / \mathrm{h})\end{array}$ & 97 & 98 & 108 & 106 & 109 & 101 & 100 & \\
\hline $\begin{array}{l}\% \mathrm{~km} \\
\text { pendiente>2\% }\end{array}$ & $2 \%$ & $8,59 \%$ & $0 \%$ & $1 \%$ & $0 \%$ & $11,46 \%$ & $1,32 \%$ & \\
\hline $\begin{array}{l}\% \mathrm{~km} \\
\text { pendiente>4\% }\end{array}$ & $0 \%$ & $0 \%$ & $0 \%$ & $0 \%$ & $0 \%$ & $8,36 \%$ & $0,22 \%$ & \\
\hline $\begin{array}{l}\text { KtCO2eq } \\
\text { totales }\end{array}$ & 813 & 2.701 & 1.533 & 2.261 & 927 & 1.308 & 4.719 & 14.283 \\
\hline $\begin{array}{l}\text { \% emisiones } \\
\text { totales RCE }\end{array}$ & $2,89 \%$ & $9,60 \%$ & $5.45 \%$ & $8,04 \%$ & $3,30 \%$ & $4,64 \%$ & $16,77 \%$ & $50,69 \%$ \\
\hline $\begin{array}{l}\text { Intensidad } \\
\text { emisiones } \\
\text { (gCO2eq/veh-km) }\end{array}$ & 306 & 315 & 287 & 261 & 259 & 260 & 257 & 275 \\
\hline \multicolumn{9}{|c|}{ CARACTERISTICAS DE LOS CORREDORES } \\
\hline $\begin{array}{l}\text { Dos } \\
\text { alternativas } \\
\text { (pago y libre } \\
\text { de pago) }\end{array}$ & $\sqrt{ }$ & $\sqrt{ }$ & $\sqrt{ }$ & $\sqrt{ }$ & $\sqrt{ }$ & $\sqrt{ }$ & $\sqrt{ }$ & \\
\hline $\begin{array}{l}\text { Corredor } \\
\text { principal } \\
\text { (IMD>20.000 } \\
\text { veh/dia) }\end{array}$ & & $\checkmark$ & $\sqrt{ }$ & $\sqrt{ }$ & & & $\sqrt{ }$ & \\
\hline $\begin{array}{l}\text { Alto \% } \\
\text { pesados } \\
(\% \mathrm{VP}>15 \%)\end{array}$ & $\checkmark$ & $\sqrt{ }$ & $\sqrt{ }$ & $\sqrt{ }$ & & & $\sqrt{ }$ & \\
\hline $\begin{array}{l}\text { Ruta } \\
\text { montañosa } \\
\text { (tramos con } \\
\text { pendientes } \\
>4 \% \text { ) }\end{array}$ & & & & & & $\checkmark$ & $\sqrt{ }$ & \\
\hline
\end{tabular}

Tabla 2 - Resultados de los corredores prioritarios

\section{ESTRATEGIAS DE REDUCCIÓN DE EMISIONES SOBRE LOS CORREDORES}

A continuación sobre los 7 corredores prioritarios identificados se aplican las estrategias de reducción de emisiones. Las estrategias de reducción se basan en la mejora de la operación e infraestructura de la carretera. Para ello se utiliza la herramienta HERA (apartado 2).

- Estrategias de operación se centran en minimizar las emisiones mediante el ajuste de la velocidad y la gestión de vehículos pesados para tener un uso eficiente de la red 
(rutas eficientes y uso efectivo de la capacidad de la carretera). El principal objetivo es tener un uso eficiente del combustible.

- Estrategias sobre la infraestructura se centran en reducir las emisiones mediante la mejora de la infraestructura (ya sea de nueva construcción en la fase de diseño) o mejora de tramos ineficientes. Por ejemplo, mediante la corrección de la alineación horizontal.

Se han creado cuatro escenarios donde se evalúan los efectos sobre las emisiones de las diferentes estrategias. Dichos escenarios se comparan con el escenario de referencia de los corredores del 2012, calculados anteriormente. Los cuatro escenarios son los siguientes:

- Escenario 1: gestión de la velocidad, se comprueban los impactos de reducir la velocidad en $-10 \mathrm{~km} / \mathrm{h}$ de los vehículos ligeros en autopistas, autovías y autovías metropolitanas sobre el de referencia.

- Escenario 2: gestión de la velocidad con vehículos pesados, se comprueban los impactos de reducir la velocidad en $-10 \mathrm{~km} / \mathrm{h}$ de los vehículos ligeros en aquellos tramos que tienen más de $15 \%$ de volumen de tráfico de vehículos pesados.

- Escenario 3: mejora del diseño de pendientes, se comprueba los impactos sobre las emisiones de tener una red sin rampas/pendientes, es decir, todos los tramos $0 \%$.

- Escenario 4: integración de las estrategias 1, 2, y 3.

En la Tabla 3 se recogen los resultados de cada escenario totales de los 7 corredores, y también los ahorros sobre el escenario de referencia y la red total RCE. Todos los escenarios producen una reducción de las emisiones GEI. Sobre los tres primeros escenarios, la gestión de la velocidad (escenario 1) es la estrategia más efectiva, con unos ahorros anuales de 3,85\% sobre los 7 corredores (566Kt CO $\mathrm{CO}_{2}$ eq de ahorro) y 2,01\% ahorros sobre el total de la RCE. Los ahorros mayores se producen en las autovías (391 Kt CO 2 eq de ahorro), dado que tienen altas velocidades y alto volumen de tráfico. En general, la reducción $-10 \mathrm{~km} / \mathrm{h}$ en autovías produce una reducción de la intensidad de emisiones en los tramos de $-10 \mathrm{~g}$ de $\mathrm{CO}_{2} \mathrm{eq} / \mathrm{veh}$ $\mathrm{km}$. Del análisis también se obtiene que la mejora del diseño de las carreteras es un elemento clave en la reducción de las emisiones en la fase de explotación (tráfico). La mejora del diseño puede producir unos ahorros del $1 \%$ sobre los siete corredores $\left(158 \mathrm{Kt} \mathrm{CO}_{2} \mathrm{eq} \mathrm{de}\right.$ ahorro). Finalmente, la integración de todas las estrategias produce unos ahorros anuales de $736 \mathrm{Kt} \mathrm{CO}_{2}$ eq de ahorro, correspondiendo con 2,62\% de ahorros sobre el total de la RCE.

\begin{tabular}{|c|c|c|c|c|}
\hline Descripción de escenarios & $\begin{array}{c}\text { Emisiones } \\
\text { GEI (Kt } \\
\text { CO2eq/año) }^{-}\end{array}$ & $\begin{array}{c}\text { Ahorros } \\
\text { Emisiones } \\
\text { GEI (Kt } \\
\text { CO2eq/año) }\end{array}$ & $\begin{array}{c}\text { Ahorros } \\
\text { Emisiones } \\
\text { GEI }(\%) \text { de } \\
\text { los siete } \\
\text { corredores }\end{array}$ & $\begin{array}{c}\text { Ahorros de } \\
\text { Emisiones GEI } \\
\text { (\%) sobre el } \\
\text { total RCE }\end{array}$ \\
\hline Escenario de referencia & 14.719 & & & \\
\hline Autopista peaje & 3.045 & & & \\
\hline Autovía & 9.046 & & & \\
\hline Carretera Nacional & 1.598 & & & \\
\hline Autovía metropolitana & 1.030 & & & \\
\hline
\end{tabular}




\begin{tabular}{|c|c|c|c|c|}
\hline Descripción de escenarios & $\begin{array}{c}\text { Emisiones } \\
\text { GEI (Kt } \\
\text { CO2eq/año) }\end{array}$ & $\begin{array}{c}\text { Ahorros } \\
\text { Emisiones } \\
\text { GEI (Kt } \\
\text { CO2eq/año) }\end{array}$ & $\begin{array}{c}\text { Ahorros } \\
\text { Emisiones } \\
\text { GEI }(\%) \text { de } \\
\text { los siete } \\
\text { corredores }\end{array}$ & $\begin{array}{l}\text { Ahorros de } \\
\text { Emisiones GEI } \\
\text { (\%) sobre el } \\
\text { total RCE }\end{array}$ \\
\hline Escenario 1: gestión velocidad & 14.153 & 566 & $3,85 \%$ & $2,01 \%$ \\
\hline Autopista peaje & 2.875 & 169 & $5,55 \%$ & \\
\hline Autovía & 8.655 & 391 & $4,32 \%$ & \\
\hline Carretera Nacional & 1.598 & 0 & $0,00 \%$ & \\
\hline Autovía metropolitana & 994 & 36 & $3,53 \%$ & \\
\hline $\begin{array}{l}\text { Escenario 2: gestión velocidad con veh. } \\
\text { pesados }\end{array}$ & 14.252 & 267 & $3,17 \%$ & $1,66 \%$ \\
\hline Autopista peaje & 3.013 & 32 & $1,04 \%$ & \\
\hline Autovía & 8.655 & 391 & $4,32 \%$ & \\
\hline Carretera Nacional & 1.797 & 0 & $0,00 \%$ & \\
\hline Autovía metropolitana & 986 & 44 & $4,27 \%$ & \\
\hline Escenario 3: mejora del diseño pendientes & 14.561 & 158 & $1,07 \%$ & $0,56 \%$ \\
\hline Autopista peaje & 3.013 & 32 & $1,05 \%$ & \\
\hline Autovía & 8.970 & 77 & $0,85 \%$ & \\
\hline Carretera Nacional & 1.576 & 22 & $1,36 \%$ & \\
\hline Autovía metropolitana & 1.002 & 28 & $2,71 \%$ & \\
\hline Escenario 4: integración estrategias & 13.983 & 736 & $5,00 \%$ & $2,62 \%$ \\
\hline Autopista peaje & 2.867 & 178 & $5,85 \%$ & \\
\hline Autovía & 8.660 & 387 & $4,27 \%$ & \\
\hline Carretera Nacional & 1.576 & 22 & $1,36 \%$ & \\
\hline Autovía metropolitana & 970 & 60 & $5,83 \%$ & \\
\hline
\end{tabular}

Tabla 3 - Resultados totales de las estrategias de reducción de emisiones sobre los 7 corredores

\section{CONCLUSIONES}

La reducción de las emisiones de tráfico es una prioridad en la planificación y gestión de infraestructuras de transporte para lograr un sistema de transporte más sostenible (SIMS et al. 2014). La metodología de evaluación de emisiones del tráfico de carreteras HERA (SOBRINO et al. 2014) combina un modelo de velocidad media ajustado con la pendiente/rampa del tramo en estudio. Utilizando HERA y la RCE de España de 2012, el presente estudio contribuye con nuevos procedimientos y políticas.

- Las ventajas de los datos georreferenciados y resultados. En el estudio se utiliza el SIG para crear una red que combina las bases de datos de tráfico y del inventario de carreteras como datos de entrada de HERA. Esto permite que las emisiones (resultados de HERA) puedan ser representadas en mapas. De esta forma, la representación tramo a tramo hace más fácil la identificación de corredores prioritarios. El uso de los datos a nivel tramo de carretera para obtener emisiones y su representación de forma vectorial, reduce la incertidumbre asociada con otros procedimientos basados en utilizar proxis (densidad carretera, población, etc.) (GURNEY et al. 2012; KINNEE et al. 2004).

- Identificación de corredores prioritarios. Para hacer frente a los problemas de 
cambio climático, es muy importante establecer un plan por orden prioridad (UNAL et al. 2004). Las estrategias deben ser aplicadas sobre las áreas que más contaminan con el objetivo de conseguir mayores ahorros. El estudio propone un índice IPE (capítulo 3) que por orden de prioridad identifica corredores con mayores problemas de emisiones. El IPE ha sido calculado para la RCE de 2012 y se representa en la Figura 3. Siete corredores prioritarios han sido identificados, que representan el $25 \%$ de la longitud de la RCE y son responsables del $51 \%$ de las emisiones GEI totales de la RCE. De los resultados se obtiene que los corredores más ineficientes son aquellos que tienen gran volumen de vehículos pesados, altas velocidades de vehículos ligeros y tramos con pendientes pronunciadas.

- Diseño de estrategias de operación e infraestructura utilizando HERA. Para la reducción de emisiones del tráfico se requieren estrategias ad-hoc. Las metodologías de evaluación de emisiones existentes normalmente se centran en diferentes fases del ciclo de vida de la carretera de forma independiente: exclusivamente en el tráfico y la operación de carreteras (GRANT et al. 2013), o en el diseño, construcción y mantenimiento de la infraestructura (TAGG, 2013). Sin embargo, se debe poner más atención en combinar los efectos de la infraestructura sobre el tráfico o viceversa. Por ejemplo, el efecto de los trabajos de mantenimiento sobre el tráfico (HUANG et al., 2009). En el presente estudio, HERA es de particular interés para el diseño de políticas y estrategias centradas en la alineación horizontal, ajuste de velocidad, gestión del tráfico, y la flota de vehículos. Puede ser utilizada tanto en la fase de diseño y planificación de la carretera (para evaluar los efectos de diferentes diseños en las emisiones de la operación) como en la explotación de la carretera. Sobre los 7 corredores identificados se han aplicado diferentes estrategias de reducción de emisiones. Los resultados muestran que la integración del ajuste de velocidad con la mejora de la alineación horizontal puede resultar en unos ahorros del 5\% en comparación con el escenario de referencia $\left(736 \mathrm{Kt} \mathrm{CO}_{2} \mathrm{eq}\right)$. La estrategia más efectiva es la reducción de velocidad sobre los vehículos ligeros en vías de alta capacidad. Una reducción de $-10 \mathrm{~km} / \mathrm{h}$ puede llegar a ahorrar $10 \mathrm{~g} \mathrm{CO} 2 \mathrm{eq} / \mathrm{veh}-\mathrm{km}$.

En conclusión, el artículo demuestra las capacidades de HERA para identificar corredores prioritarios mediante el uso de SIG y el desarrollo de un índice basado en las emisiones totales y la intensidad de emisiones por tramo. Para las futuras investigaciones se debería realizar un análisis exploratorio sobre los factores más influyentes de la huella de carbono del tráfico. También sería de interés que sobre los resultados de emisiones por tramo de la RCE obtenidos del presente análisis, se obtuviese un índice del nivel de eficiencia de emisiones por tramo (similar al nivel de servicio por tramo) con el objetivo de medir la eficiencia de emisiones de los tramos dados un volumen de tráfico y diseño establecidos. Finalmente, otro aspecto a considerar sería la introducción de este procedimiento en un sistema de toma de decisiones para medir todos los impactos territoriales de la infraestructura del transporte por carretera (ORTEGA et al. 2014) con el objetivo de incorporar los impactos de emisiones en la evaluación medioambiental (ARCE y GULLÓN, 2000; ARCE et al. 2010). 


\section{AGRADECIMIENTOS}

Los autores agradecen a Pablo Pérez de Villar por proporcionar los datos de la RCE. Natalia Sobrino agradece también a la Universidad Politécnica de Madrid (UPM) por la beca de investigación concedida (2011-2015) que le ha permitido desarrollar este trabajo de investigación enmarcado en los estudio de su tesis doctoral "Methodologies to measure and to manage the decarbonisation of road transport. The case of Spain", 2015.

\section{REFERENCIAS}

AEMA (2014). Why did greenhouse gas emissions decrease in the UE between 1990 and 2012? Disponible en: <http://www.eea.europa.eu/publications/why-are-greenhousegasesdecreasing $>$ [Acceso 25 Sep. 2014].

ARCE, R.; GULLÓN, N. (2000) The application of Strategic Environmental Assessment to sustainability assessment of infrastructure development. Environmental Impact Assessment Review, 20(3), pp. 393-203.

ARCE, R.; ORTEGA, E.; OTERO, I. (2010) Los sistemas de información geográfica aplicados a la evaluación ambiental en la planificación de las infraestructuras del transporte. Ciudad y Territorio. Estudios Territoriales, 42(165-16), pp.513-528.

BANISTER, D.; ANDERTON, K.; BONILLA, D.; GIVONI, M.; SCHWANEN, T. (2011) Transportation and the Environment. Annual Review of Environment and Resources, 36(1), pp.247-270.

COM (2010) Comunicación de la Comisión al Parlamento Europeo, al Consejo, al Comité Económico y Social Europeo y al Comité de las Regiones: Energía 2020: estrategia para una energía competitiva, sostenible y segura (COM 2010639 final de 10.11.2010). Disponible en: <http://eur-lex.europa.eu/legal-content/ES/TXT/?uri=URISERV:en0024> [Acceso 28 Feb. 2016].

DGT (2013) Anuario estadístico 2012. Online: <http://www.dgt.es/es/seguridadvial/estadisticas-e-indicadores/parque-vehiculos/series-historicas/> [Acceso 28 Feb. 2016].

EUROSTAT (2014) Greenhouse gas emissions by sector. [Base de datos] Disponible en: <http://epp.eurostat.ec.europa.eu/portal/page/portal/environment/data/main_tables> [Acceso 16 Jun. 2014].

GKATZOFLIAS, D.; KOURIDIS, C.; NTZIACHRISTOS, L; SAMARAS, Z. (2007) COPERT 4: Computer Program to Calculate Emissions from Road Transport. (Online) Laboratory of Applied Thermodynamics, Mechanical Engineering Department of Aristotle University Thessaloniki; and European Environment Agency. Available: http://emisia.com/products/copert-4 [Acceso 28 Feb. 2016].

GRANT, M.; HARTLEY, W.S.; MILAM, R.; WALTERS, J. et al. (2013) Handbook for estimating transportation greenhouse gases for integration into the planning process. Technical report FHWA-HEP-13-026. Online: https://www.fhwa.dot.gov/environment/climate_change/mitigation/publications/ghg_hand 
book/index.cfm> [Acceso 28 Feb. 2016].

GURNEY, K.R.; RAZLIVANOV, I.; SONG, Y.; ZHOU, Y. et al. (2012) Quantification of fossil fuel CO2 emissions on the building/street scale for a large U.S. City. Environmental Science and Technology, 46(21), 12194-12202.

HERNANDEZ, S.; SOBRINO, N.; MONZON, A. (2013) Carbon footprint of light vehicles: road gradient effect. En: I Jornada sobre Vehículos y Transportes: I+D+I en la UPM. Fundación General de la Universidad Politécnica de Madrid.

HUANG, Y.; BIRD, R.; BELL, M. (2009) A comparative study of the emissions by road maintenance works and disrupted traffic using life cycle assessment and micro-simulation. Transportation Research Part D: Transport and Environment, 14(3), pp. 197-204.

IDAE (2003) Estrategia de Ahorro y Eficiencia Energética en España 2004-2012 (E4). Disponible

en: $<$ http://idae.electura.es/publicacion/8/estrategia_ahorro_eficiencia_energ\%EF\%BF\%BDtic a_espa\%EF\%BF\%BDa_2004-2012_e4> [Acceso 28 Feb. 2016].

IDAE (2011) Segundo Plan de Acción Nacional de Eficiencia Energética en España 2011 2020. Disponible en:

<http://www.idae.es/index.php/mod.documentos/mem.descarga?file=/documentos_11905_ PAEE_2011_2020._A2011_A_a1e6383b.pdf> [Acceso 28 Feb. 2016].

KINEE, E.J.; TOUMA, J.S.; MASON, R.; THURMAN, J. et al. (2004) Allocation of onroad mobile emissions to road segments for air toxics modelling in an urban area. Transportation Research Part D: Transport and Environment, 9(2), pp. 139-150.

MF (2010) Inventario de la red de carreteras del estado, España. Online: $<$ http://www.fomento.gob.es/MFOM/LANG_CASTELLANO/DIRECCIONES_GENERA LES/CARRETERAS/CATYEVO_RED_CARRETERAS/INVENTARIO/> [Acceso 28 Feb. 2016].

MF (2013) Mapa de tráfico de España 2012. Online: $<$ http://www.fomento.gob.es/MFOM/LANG_CASTELLANO/DIRECCIONES_GENERA LES/CARRETERAS/TRAFICO_VELOCIDADES/MAPAS/SERIE_HISTORICA/2012/> [Acceso 28 Feb. 2016].

ORTEGA, E.; OTERO, I.; MANCEBO, S. (2014) TITIM GIS-tool: A GIS-based decision support system for measuring the territorial impact of transport infrastructures. Expert Systems with Applications, 41(16), pp. 7641-7652.

RUIZ-PADILlO, A.; TORRIJA, A.J.; RAMOS-RIDAO, A.; RUIZ, D.P. (2014) A methodology for classification by priority for action: Selecting road stretches for network noise action plans. Transportation Research Part D: Transport and Environment, 2966-78.

SIMS, R., SCHAEFFER, R., CREUTZIG, F., ET AL. (2014). Transport. En: Climate Change 2014: Mitigation of Climate Change. Contribution of Working Group III to the Fifth Assessment Report of the Intergovernmental Panel on Climate Change. Cambridge, UK and NY, USA. 
SOBRINO, N.; MONZON, A.; HERNANDEZ, S. (2014) Reduced Carbon and Energy Footprint in Highway Operations: The Higway Energy Assessment (HERA) Methodology. Networks and Spatial Economics, pp.1-20.

TAGG (2013) Greenhouse gas assessment workbook for road project. Online: < http://www.rms.nsw.gov.au/documents/about/environment/greenhouse-gas-assessmentworkbook-road-projects.pdf> [Acceso 28 Feb. 2016].

UNAL, A.; FREY, H.C.; ROUPHAIL, N.M. (2004) Quantification of highway vehicle emissions hot spots based upon on-board measurements. Journal of the Air and Waste Management Association, 54(2), pp. 130-140. 